\title{
Proyecto, desarrollo y valoración en la UPC de un caso de feedforwarding en campus virtual
}

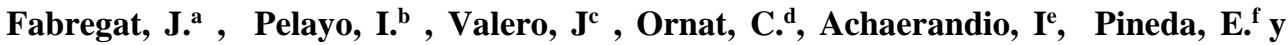
Buenestado, $\mathbf{p}$

a Profesor de matemática aplicada, Escola Superior d'Agricultura de Barcelona (UPC), jaime.fabregat@upc.edu,, bProfesor de matemática aplicada, Escola Superior d’Agricultura de Barcelona (UPC), ignacio.m.pelayo@upc.edu, 'Profesor de estadística, Escola Superior d’Agricultura de Barcelona (UPC), jordi.valero@upc.edu , d'Subdirector de TIC e infraestructuras, Escola Superior d'Agricultura de Barcelona (UPC), cesar.ornat@upc.edu, e'Coordinadora del Grup d' interès en l'ús de MOODLE-ATENEA (UPC), maria.isabel.achaerandio@upc.edu, fJefe de Estudios, Escola Superior d’Agricultura de Barcelona, eloi.pineda@upc.edu y gProfesor de matemática aplicada, Escola Universitària d’Enginyeria Tècnica Industrial de Barcelona (CEIB), pablo.buenestado@upc.edu

\begin{abstract}
Several professors of UPC, UB, UAB, UIB, UOC and URV are investigating on "feed forwarding", a formative evaluation which is fed back immediately, which is not limited to "right-wrong", which is guiding rather than resolving, which is sufficiently individualized, which motivates reflection - action and which garantees a sustainable task for students and professors.

The application inside the UPC has fallen on "Mathematics" at the College of Agriculture of Barcelona, during the first semester. It has designed an experience transferable to more topics, using props NTIC, and it has analyzed the level of achievements resulting from their deployment. To encourage learning, some questionnaires about the discipline were sent out, with and without feedback. Students have to first consider a feedback questionnaire, repeatedly until they have successfully answered all the questions, and then have faced a similar questionnaire without feedback.

The experience has carried out involving two hundred students, with five double questionnaires generated by means of WIRIS (corresponding to the five themes of the course), using the Moodle 2.6.2. We have studied the contribution to academic achievement and satisfaction of agents, confirming increased dedication to matter, improvements (not maintained) in learning and positive evaluation of the step taken.
\end{abstract}

Keywords: assessment, feedback, feedforwarding, mathematics 
Proyecto, desarrollo y valoración en la UPC de un caso de feedforwarding en campus virtual

\begin{abstract}
Resumen
Docentes de UPC, UB, UAB, UIB, UOC y URV investigamos sobre una evaluación formativa "feedforwarding", que realimente de inmediato, que no se limite al "correcto-incorrecto", que guíe más que resuelva, que sea suficientemente individualizada, que motive reflexión - acción y que genere un trabajo sostenible para alumnos y profesores.
\end{abstract}

La aplicación en la UPC ha recaído sobre "Matemáticas" del primer cuatrimestre en la Escuela Superior de Agricultura de Barcelona. Se ha diseñado una experiencia transferible a más asignaturas, que usa apoyos de NTIC y que permite analizar eventuales logros resultantes de su despliegue. Para fomentar el aprendizaje se han administrado cuestionarios sobre la disciplina, con y sin realimentación. Los estudiantes han considerado primero un cuestionario con realimentación reiterada hasta que responden bien todas las preguntas $y$, posteriormente, han afrontado un cuestionario similar sin realimentación.

Se ha actuado sobre unos doscientos estudiantes, con cinco cuestionarios dobles generados con WIRIS (correspondientes a los cinco temas de la asignatura), empleando la versión Moodle 2.6.2. Se ha estudiado la contribución al rendimiento académico y la satisfacción de los agentes, constatándose aumento de dedicación a la materia, mejoras (no duraderas) en el aprendizaje y valoración positiva del paso dado.

Palabras clave: evaluación, feedforwarding, matemáticas, realimentación

\title{
1. Introducción
}

La perspectiva de la filosofía de la educación implica, epistemológicamente, poner entre paréntesis la cüestión del "tener que hacer”, para introducir la pregunta sobre "qué es mejor" (Pagés, 2013)

La evaluación es un tema clave, pero también una asignatura pendiente en el campo educativo (Bartolomé, 2012). Dentro de los planes de estudios, el alumno sigue percibiendo la evaluación como calificadora (que permite aprobar) más que como formativa (que ayuda a aprender); incluso no pocas veces se la presenta únicamente como acreditativa. Sin embargo, es la faceta formativa de la evaluación la que resulta clave para el aprendizaje. Es, por tanto, la faceta que esencialmente ha de ser planteada en la universidad por su valor y de la que cabe esperar, de modo permanente, mayores y mejores resultados a tenor de una aplicación consistente de la misma. 
Fabregat, J., Pelayo, I. , Valero, J, Ornat, C. , Achaerandio,I, Pineda, E. y Buenestado, P.

En la evaluación formativa, un plan que incluya explícitamente realimentación, "feedback", desempeña un papel necesario, de enorme relevancia e impacto, dado que puede suministar información, clarificar, flexibilizar métodos y tiempos, favorecer impactos, proporcionar oportunidades de autoevaluación, facilitar en el estudiante la reflexión sobre el propio trabajo, estimular el diálogo y otras interacciones, dar apoyos, espolear la autoestima, motivar, animar, implicar a los estudiantes Dentro del marco amplio de evaluación con posibilidades de realimentación es viable decantarse por una alternativa de secuencia de acciones "feedforwarding". Para su análisis, unos criterios que constituyen una pauta son los de selectividad, pertinencia, orientación hacia los resultados, contextualización, capacidad de transferencia, equilibrio y fomento de la reflexión (Nicol, 2007).

El desarrollo experimentado por las tecnologías de la información y comunicaciones (TIC) en las tres últimas décadas fundamenta, y justifica, que se abran vías nuevas de investigación teórica y aplicada, a las que añadiendo el progreso tecnológico produzcan como resultado la obtención de unas herramientas, basadas en el uso de ordenadores, que puedan ser presentadas y utilizadas para poner en marcha públicamente proyectos. Estos deben elevar el rendimiento del proceso educativo, aumentar la capacidad de autoaprendizaje del estudiante proporcionándole evidencias neutrales de sus logros (junto con un análisis de tales evidencias), facilitar la autoevaluación de sus conocimientos y evaluar también el proceso enseñanza/aprendizaje en el que están inmersos tanto el estudiante como el profesor (Fernández Sánchez et al., 2009)

La atención verdaderamente proporcionada al estudiante mediante la generación de aplicaciones más conscientes de las necesidades de la persona, reaccionando de manera inteligente y autónoma a las mismas, mejora la experiencia del estudiante dentro del ámbito educativo (Martín et al., 2010)

\section{Objetivos}

El proceso aquí explicado tiene como objetivo inmediato colaborar con una actividad evaluadora integrada en el procedimiento de calificación, para que se convierta en una actividad evaluadora que sea clara, dinámica y fundamentalmente formativa. Se trata de ayudar a cada estudiante, de forma individualizada, a que se percate de sus errores, de probables causas de los mismos, así como de caminos para salir de ellos. Se trata también de ayudar a cada estudiante a enfrentarse a retos con honestidad, facilitándole una guía para que vaya superándolos responsablemente. Y se trata de hacerlo todo con cuidado, pero al mismo tiempo con rapidez para evitar tiempos muertos en la preparación del estudiante, y se trata de hacerlo de manera que los profesores puedan atender simultáneamente a numerosos estudiantes de niveles muy heterogéneos.

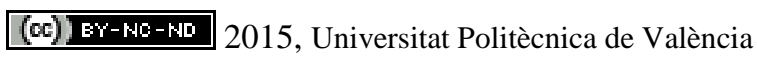

Congreso In-Red (2015) 
Proyecto, desarrollo y valoración en la UPC de un caso de feedforwarding en campus virtual

Una encuesta final solicitará a los estudiantes una valoración personal con variables cuantitativas, así como un comentario del proceso, de su forma de trabajo y de sus resultados en la asignatura. Otra encuesta pedirá también el sentir de los profesores por medio de un instrumento que permita conocer su percepción general, y sobre aspectos específicos, de la experiencia realizada de "feedforwarding". Entre tales aspectos específicos cabe citar el tipo de "feedforwarding", los agentes, las competencias promovidas y los centros de atención del "feedforwarding” aplicado.

\section{Desarrollo de la innovación}

El ensayo para alcanzar los objetivos descritos en el apartado anterior se ha aplicado a dos centenares de estudiantes universitarios de matemáticas de primer cuatrimestre en la Escola Superior d'Agricultura de Barcelona, que cursan titulaciones en ingeniería de biosistemas. Se han administrado dos modalidades de unos cuestionarios de preguntas sobre la disciplina (accesibles desde el ordenador), que han motivado que se programe y se aplique un esfuerzo complementario destinado al aprendizaje, ante el que la responsabilidad de estudiantes y profesores ha dado su "sí". La experiencia se ha realizado dentro del proyecto REDICE-14 (UB) "Diseño, implementación y evaluación de feedforwarding”, que reúne iniciativas varias desplegadas por equipos de diversos departamentos y centros universitarios, los cuales desarrollan innovación e investigación en el área educativa.

Se ha partido de respetar la premisa incorporada en la praxis de la asignatura, que se basa en consideraciones teóricas y contextuales (y no exenta de pragmatismo), de que no es necesario (quizá tampoco provechoso) garantizar de modo inexorable que se presuponga que cada estudiante afronte solo determinadas evaluaciones, pero que sí procede asegurar que sea difícil llevar a cabo prácticas de parasitismo estéril. De acuerdo con esto se han preparado cinco cuestionarios dobles con preguntas sobre la materia, no idénticas para todos los estudiantes, aunque sí similares, contándose para su generación con las potencialidades de WIRIS, para lo que el profesorado de la asignatura ha recabado y obtenido una formación específica desde los cursos para docentes del Instituto de Ciencias de la Educación.

\subsection{Cuestionarios}

Se ha vinculado con cada uno de los cinco bloques temáticos de la asignatura (álgebra lineal, ...) un cuestionario doble, con uno de los modelos de carácter "adaptativo" - con realimentación - y el otro de carácter "estándar” - sin realimentación - . La mayoría de las preguntas se han confeccionado sobre la base de un formato de ejercicio, aunque también hay algunas con un planteamiento más teórico. Los cuestionarios se han distribuido al concluir los desarrollos de los bloques correspondientes. 
Fabregat, J., Pelayo, I. , Valero, J, Ornat, C. , Achaerandio,I, Pineda, E. y Buenestado, P.

Se ha pedido a cada estudiante que conteste privadamente los cuestionarios mediante conexión por red a "Atenea", que es el campus virtual de la UPC basado en Moodle (actualmente Moodle 2.6), adaptado por UPCNet. Puede obrar donde sea y cuando quiera (en una franja temporal de 48 horas, que va desde las dos de la tarde de un jueves hasta la misma hora del sábado siguiente). Mientras el estudiante intenta responder las preguntas puede ir recurriendo a todo tipo de ayudas, pero se promueve que actue siempre a título individual y bajo su propio punto de vista.

La iniciativa ha incorporado que para cada cuestionario, de respuesta múltiple con cuatro opciones, el estudiante trabaje primero sobre una versión que multirealimenta formativamente cuando se cometen errores (y que también "aplaude” los aciertos)

Las realimentaciones aplicadas tras las respuestas incorrectas tratan de mostrar la inadecuación "per se" de las mismas (por su "sin sentido", incoherencia, carácter desorbitado de su valor, ...) y/o bien tratan de mostrar un camino que ayude a encontrar la respuesta correcta. Las realimentaciones comentan aspectos importantes (criterio de selectividad), hacen referencia a objetivos de aprendizaje derivados de la tarea (criterio de pertinencia), mencionan resultados de aprendizaje vinculados al quehacer (criterio de orientación hacia los resultados), indican cuestiones de aplicación no singulares sino trasladables (criterio de transferibilidad), aluden tanto a aspectos positivos como negativos vistos en la tarea (criterio de equilibrio) y enfocan procesos autoreguladores (criterio de fomento de la reflexión).

Otro criterio también se verifica cuando en una encuesta final de satisfacción se invita al estudiante a meditar sobre su entrega a la tarea: se trata del criterio de compromiso. Los textos de las realimentaciones son redactados para que se refieran y busquen en el futuro introducir mejoras en sus tareas.

En cuanto a aspectos formales cabe decir que en la heteroevaluación las palabras utilizadas para las realimentaciones son respetuosas siempre, amigables o neutras, jamás agresivas y/o desagradables. Se ha buscado también la corrección lingüística y la estructura en la forma, así como un estilo que tenga un carácter convincente y que sea capaz de sugerir cambios a mejor a partir de las propias equivocaciones, evitando por otro lado dictar consignas. Las realimentaciones son de expresión abierta, no aportan las respuestas correctas sino que guían, dan pautas al estudiante para que sea él mismo quien las consiga.

En lo que corresponde a vertientes emocionales, las realimentaciones prescinden de comprimir la autoestima - exhortando ímplícitamente a los estudiantes a obtener partido de si mismos - , patrocinan una modulación de la asertividad (usando el modo condicional y/o entradas manejables más suaves que duras) y son motivadoras (alientan a conquistar avances).

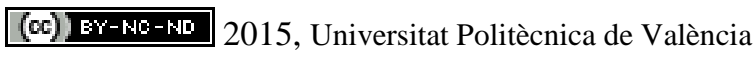

Congreso In-Red (2015) 
El estudiante recibirá posteriormente, de forma separada, una versión nueva, esta vez de tipo convencional, semejante a la originaria aunque sin realimentación, pero de ningún modo con anterioridad a haber conseguido responder correctamente todas las preguntas de la versión inicial.

Será exclusivamente de la última versión de donde se extraigan los datos que se incorporen a la calificación. De la primera versión se conseguirá de la misma manera un anchuroso volumen de datos, pero únicamente para manifestar la contribución de esta forma de desenvolverse en la consecución de aprendizajes. Con objeto de responder cada una de las versiones de los cuestionarios, el tiempo máximo entre apertura y cierre se ha fijado en dos horas.

De lo que antecede, los estudiantes de la asignatura de matemáticas involucrada en la experiencia han sido convenientemente informados con anterioridad a la puesta en marcha del proceso que les implica, siendo conocedores por lo tanto de los objetivos que se persiguen, de la forma de desarrollar las actuaciones y de la manera de sacar provecho del mismo.

En cuanto a la configuración de los cuestionarios cabe decir que:

- La versión actual de Moodle permite configurar las preguntas con retroacción especifica para la respuesta a la pregunta, y de forma general una retroacción para respuestas correctas, parcialmente correctas o incorrectas.

- A escala de cuestionario, Atenea-Moodle, permite tambien configurar la retroacción global en función de la calificación del cuestionario.

- También al definir el cuestionario unos parámetros concretos permiten determinar el momento en el que el estudiante tiene acceso a la revisión del cuestionario, así como también a las diferentes retroacciones configuradas a nivel de pregunta o a retroacción global en el cuestionario.

- Al configurar el cuestionario, también se puede configurar el número de intentos y tiempo que debe pasar entre dos intentos consecutivos del mismo cuestionario.

\subsection{Ejemplos de preguntas}

A continuación se ponen de manifiesto algunos ejemplos de cuestiones extraidas del depósito general básico de propuestas de preguntas, conteniendo asimismo las realimentaciones consignadas para cada opción de respuesta, las cuales son aquellas que se ponen a la vista justo inmediatamente después de que el estudiante se haya decantado por esta determinada alternativa. 
Fabregat, J., Pelayo, I. , Valero, J, Ornat, C. , Achaerandio,I, Pineda, E. y Buenestado, P.

\subsubsection{Geometría y trigonometría en el plano}

Los requerimientos que han de verificar dos circunferencias són: a) ser tangentes ; b) el centro de una de las mismas ha de ser uno de los puntos de la otra ; c) la mayor tiene que poseer un diámetro de valor 10 ; d) una ha de estar centrada en el origen. Una ecuación para una de las circunferencis puede ser:

(A) $\mathrm{x}^{2}+\mathrm{y}^{2}=50 \quad$ El radi $\sqrt{ } 50 \quad$ no és el de la circunferencia mayor (5) ni el de la circunferencia menor (que debería ser un valor más reducido todavía)

(B) $\mathrm{x}^{2}+\mathrm{y}^{2}=6,25$ Efectivamente, ésta es la respuesta correcta

(C) $\mathrm{x}^{2}+\mathrm{y}^{2}=100$ ¿Has usado el diámetro de una circumferència como si fuese su radio?

(D) $\mathrm{x}^{2}+\mathrm{y}^{2}=2,5$ La ecuación de la circunferencia de centro $(0,0)$ y radio $r$ és $x^{2}+y^{2}=\boldsymbol{r}^{2}$. Resulta probable que en el segundo miembro se haya procedido a registrar el radio de la circunferencia en lugar de su cuadrado

\section{Ejemplo 1}

De las gráficas que corresponden a las siguientes tres ecuaciones

a) $4 x^{2}-9 x+y-5=0$

b) $4 x^{2}-y^{2}+8 x-6 y+4=0$

c) $2 x^{2}+4 y^{2}-4 x+12 y=0$

(A) Sólo hay una que sea una parábola de eje vertical

Esta es la respuesta correcta

(B) Hay exactamente dos que son circunferencias

¿Has pensado que las ecuaciones de segundo grado de dos variables corresponden a circunferencias? Hay que exigir además unos cuadrados multiplicados por lo mismo

(C) Hay sólo una que es una circunferencia

Tal vez has pensado que todas las ecuaciones de segundo grado de dos variables donde sale $x^{2}$ y también $y^{2}$, ambos con coeficientes del mismo signo, son circunferencias: no es cierto ya que hay que exigir que ambos coeficientes sean iguales

(D) Todas son parábolas

Tal vez has pensado que todas las ecuaciones de segundo grado de dos variables que no dan circunferencias corresponden a parábolas: no es cierto (pueden salir otras líneas)

Ejemplo 2

(cc) EY-NC-ND 2015, Universitat Politècnica de València

Congreso In-Red (2015) 
En una circunferencia de radio 2 y centro $(0,0)$, asociamos a cada real $r$ un punto de la circunferencia. Partiendo de $(2,0)$ se recorre una longitud $r$ en sentido antihorario si $r>0$ o en sentido horario, si $r<0$. Si para un real $r$ el punto de la circunferencia es $(x, y)$ entonces

(A) sen $\mathrm{r}=$ y para toda $\mathrm{r}$ real

Esto sería cierto sólo si la circunferencia fuese unitaria.

(B) $\csc \mathrm{r}=2 / \mathrm{y}$ para toda $\mathrm{r}$ real

Esto no es cierto en los puntos $k \pi$ ( $k$, entero)

(C) $\cos \mathrm{r}=\mathrm{x} / 2$ sólo para los reales diferentes de $(2 \mathrm{k}+1) \pi / 2$ ( $\mathrm{k}$, entero)

Esto es verdadero para cualquier número real.

(D) $\tan \mathrm{r}=\mathrm{y} / \mathrm{x}$ sólo para los reales diferentes de $(2 \mathrm{k}+1) \pi / 2(\mathrm{k}$, entero)

He aquí la respuesta correcta

Ejemplo 3

\subsubsection{Funciones reales de variable real}

Derivando $\mathrm{f}(\mathrm{x})=$ xarctan $\mathrm{x}$ sale

(A) $1 /\left(1+x^{2}\right)$

¿No será que has pensado que "derivada de producto" es "producto de derivades"?

$(B) \tan x+\left[x\left(1+\tan ^{2} x\right)\right]$

$¿$ No has acaso confundido arctanx con tanx?

(C) $\arctan x-\operatorname{xcsc}^{2} \mathrm{x}$

¿No habrá ocurrido que hayas considerado que arctanx $=1 / \tan x$ ?

(D) $\arctan x+\left[\mathrm{x}\left(1+\mathrm{x}^{2}\right)\right]$

Esta es, en efecto,la respuesta correct

Ejemplo 4 
Fabregat, J., Pelayo, I. , Valero, J, Ornat, C. , Achaerandio,I, Pineda, E. y Buenestado, P.

Sea la función $\mathrm{f}(\mathrm{x})$ tal que $\mathrm{f}(\mathrm{x})=1-\mathrm{x}^{2}-4 \mathrm{x}$ si $\mathrm{x}<-1, \mathrm{f}(\mathrm{x})=\mathrm{x}-1$ si $-1<\mathrm{x}$. Su gràfica
(A)es creciente
En el tramo de la izquierda es un arco de parábola con una zona creciente y una decreciente
(B)tiene un tramo horizontal
No puede tener ningún tramo horizontal puesto que no es una función constante en ningún
tramo
(C)tiene un tramo coincidente con la bisectriz del primer cuadrante
Tiene un tramo paralelo a la bisectriz del primer cuadrante pero no coincidente con ella
(D)tiene un tramo rectilíneo de pendiente 1
Esta afirmación es la correcta. El tramo rectilíneo es el de la derecha.

Ejemplo 5

\section{Resultados}

Se acredita que los estudiantes han respondido los cuestionarios dobles (a pesar de la no estricta obligatoriedad: el peso global en la calificación era sólo del $5 \%$, un $1 \%$ por cuestionario); lo han hecho con unos porcentajes que han superado siempre el $50 \%$ y rozado el $80 \%$ en la mayoría de casos. En cifras absolutas, en un momento u otro han llegado a estar involucrados en la experiencia 212 estudiantes (de un total de 243 matriculados).

Con objeto de estudiar la formación conseguida con el método, en cada aplicación de cuestionarios, para cada pregunta se ha realizado un test de proporciones uniformes y se han determinado intervalos de confianza de proporciones (no acumuladas y acumuladas) en diferentes supuestos; al mismo tiempo se han aplicado pruebas de Chi-cuadrada.

Del estudio estadístico se extrae que la elección de respuesta en los dos primeros intentos (hay cuatro opciones posibles) no se efectua al azar; en cambio en cuanto a la elección en el tercer intento, en general ya no hay evidencia de no aleatoriedad.

Las calificaciones alcanzadas en el cuestionario aportador de nota han sido de calidad alta: de forma rotundamente mayoritaria entre el notable y el excelente, con deriva hacia este último. Sin embargo estos resultados no han tenido su correspondiente reflejo en las calificaciones finales de la asignatura.

La única ligazón percibida a tenor del estudio estadístico entre datos vinculados a la experiencia y calificaciones finales es que éstas mantienen una relación con el número de cuestionarios (dobles) contestados: se constata en el sentido natural de que a más cuestionarios (dobles) respondidos más alta calificación final se consigue y que a menos cuestionarios (dobles) respondidos menor calificación final se. alcanza Concretando en mayor medida, se puede decir que la calificación final de los que han hecho los cinco

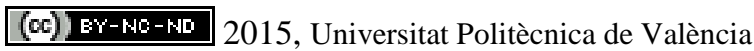

Congreso In-Red (2015) 
cuestionarios dobles (es decir todos) es más alta que la de todos los demás, y la de los que han hecho cuatro es más alta que la de los que no han hecho ninguno.

Por otro lado, todas las calificaciones del cuestionario del modelo"adaptativo" - cuestionario con realimentación - estan muy relacionadas con las del modelo "estandard" - cuestionario sin realimentación - . La calificación más relacionada con la del modelo "estandard" es la que toma sólo en cuenta la primera respuesta dada en el cuestionario del modelo "adaptativo".

Para refrendar la percepción de los agentes se han distribuido encuestas de satisfacción a los promotores / gestores y a los usuarios. Entrevistas a estudiantes han comunicado, en forma desarrollada , como se han situado ellos mismos ante la experiencia.

Los indicadores del grado de satisfacción con la experiencia han mostrado un nivel alto por parte de todos los agentes, aunque gestar los cuestionarios haya exigido a los profesores un tiempo largo de dedicación complementaria.

Se encuentra pendiente de realización- difusión un análisis más profundo de los cuestionarios de satisfacción administrados a estudiantes y profesores. Se ha pospuesto su examen a fondo a que haya avanzado más el conjunto de despliegues que llevan a cabo las seis universidades implicadas en el proyecto.

\section{Conclusiones}

La evaluación aplicada ha obtenido un alto seguimiento por los estudiantes (que ha llegado en un momento u otro a albergar al $87 \%$ de los matriculados), sin que éstos hayan planteado objeciones, aun pidiéndoles un tiempo, un esfuerzo y una reflexión considerables (si bien no desmesurados). En el modelo de cuestionario de opción múltiple con realimentación los estudiantes no han respondido por puro azar en ninguno de los dos primeros intentos: ello significa que han puesto interés y atención (y un cierto nivel de conocimiento).

La calificación final y la cantidad de cuestionarios respondidos no se privan de ser concomitantes. Tampoco se privan las notas de los controles del modelo "adaptativo" de estar muy conectadas con las del modelo "estandard": las notes más conectadas son las notas derivades de tomar en consideración sólo las respuestas aportades en el primer intento. En cambio, apenas se observa que las notss de los cuestionarios contribuyan a explicar las notas finales.

El trabajo de ir confeccionando los cuestionarios, en lo que concierne al banco de preguntas y de realimentaciones y también de generación de variantes con estrategia, ha exigido una elevada dedicación al profesorado, pero la labor ya efectuada permitirá próximas utilizaciones del sistema formativo con un coste más reducido. 
Fabregat, J., Pelayo, I. , Valero, J, Ornat, C. , Achaerandio,I, Pineda, E. y Buenestado, P.

El poco tiempo entre responder un cuestionario muy guiado y otro no igual pero sí similar, eventuales acciones de picaresca (aunque han querido ser evitadas y se ha actuado para que así fuera), la inexistencia explícita (y también implícita) de límites a un uso de documentación de apoyo (sobre papel o vista en pantalla), así como la ausencia de restricciones en el empleo de software matemático de menor o mayor vuelo, pueden justificar que los grados de éxito percibidos en la respuesta a los cuestionarios no concuerden con en el aprendizaje global demostrado en conjunto, traducido en unas calificaciones finales de la asignatura claramente inferiores a las esperadas. Entrevistas con estudiantes, a modo de grupo de discusión, han aportado luz sobre este tema: ello permitirá a los profesores reorientar ediciones posteriores con más conocimiento de causa.

\section{Referencias}

BARTOLOMÉ PINA, A. (2012). “Prólogo” en Cano García, E. (ed.) Aprobar o aprender. Estrategias de evaluación en la sociedad red. Barcelona : Laboratori de Mitjans Interactius. Universidad de Barcelona

FERNÁNDEZ SÁNCHEZ, P., et al. (2009). "El aprendizaje activo mediante la autoevaluación utilizando un laboratorio virtual" en IEEE-RITA Revista Iberoamericana de Tecnologías del Aprendizaje, vol. 4, num. 1, p. 53-62

MARTín, S., et al. (2010). "M2Learn: Framework Abierto para el Desarrollo de Aplicaciones para el Aprendizaje Móvil y Ubicuo" en IEEE-RITA Revista Iberoamericana de Tecnologías del Aprendizaje, vol. 5, num. 4, p. 138-145

NICOL, D. (2007). « Principles of good assessment and feedback : Theory and practice » En REAP International Online Conference on Assessment Design for Learner Responsibility. Disponible en http://www.reap.ac.uk/reap07/Portals/2/CSL/keynotes/david\%20nicol/Principles_of_good_assessmen t_and_feedback.pdf [Consulta: 2013, 15 de mayo].

PAGÉS SANTACANA, A. (2013). « Tecnologia i lideratge educatiu : una perspectiva filosòfica » en Aloma, Revista de Psicologia, Ciències de l'Educació i de l'Esport, vol. 31, num. 1, p. 15-22.

(cc)) EY-NC-ND 2015, Universitat Politècnica de València

Congreso In-Red (2015) 